Journal for ImmunoTherapy of Cancer

\title{
Pretreatment metastatic growth rate determines clinical outcome of advanced melanoma patients treated with anti-PD-1 antibodies: a multicenter cohort study
}

Nikolaus B Wagner (D) , ${ }^{1,2}$ Max M Lenders, ${ }^{1}$ Kathrin Kühl, ${ }^{3,4}$ Lydia Reinhardt,, ${ }^{3,4}$ Fiona André, ${ }^{5}$ Milena Dudda, ${ }^{6}$ Natalie Ring, ${ }^{7}$ Chiara Ebel, ${ }^{8}$ Ramon Stäger, ${ }^{9}$ Caroline Zellweger, ${ }^{10}$ Roland Lang, ${ }^{11}$ Michael Paar, ${ }^{12}$ Philipp Gussek, ${ }^{13}$ Georg Richtig, ${ }^{14,15}$ Suzan H Stürmer, ${ }^{16}$ Susanne Kimeswenger, ${ }^{17}$ Angela Oellinger, ${ }^{17}$ Andrea Forschner (D) , ${ }^{1}$ Ulrike Leiter, ${ }^{1}$ Benjamin Weide, ${ }^{1}$ Maximilian Gassenmaier, ${ }^{1}$ Amadeus Schraag, ${ }^{18,19}$ Bernhard Klumpp, ${ }^{19,20}$ Wolfram Hoetzenecker, ${ }^{17}$ Carola Berking, ${ }^{16,21}$ Erika Richtig, ${ }^{15}$ Mirjana Ziemer, ${ }^{13}$ Johanna Mangana, ${ }^{9}$ Patrick Terheyden (D) , ${ }^{8}$ Carmen Loquai, ${ }^{6}$ Van Anh Nguyen, ${ }^{5}$ Christoffer Gebhardt (1) ,22 Friedegund Meier, ${ }^{3,4}$ Stefan Diem, ${ }^{23}$ Antonio Cozzio, ${ }^{2}$ Lukas Flatz, ${ }^{1,2}$ Martin Röcken, ${ }^{1}$ Claus Garbe, ${ }^{1}$ Thomas K Eigentler ${ }^{1}$

\section{ABSTRACT}

Background Checkpoint inhibitors revolutionized the treatment of metastatic melanoma patients. Although tumor burden and lactate dehydrogenase (LDH) are associated with overall survival (OS), the impact of tumor growth kinetics remains elusive and in part contradictory. The aims of this study were to develop a novel simple and rapid method that estimates pretreatment metastatic growth rate (MGR) and to investigate its prognostic impact in melanoma patients treated with antiprogrammed death receptor-1 (PD-1) antibodies.

Methods MGR was assessed in three independent cohorts of a total of 337 unselected consecutive metastasized stage IIIB-IV melanoma patients (discovery cohort: $n=53$, confirmation cohort: $n=126$, independent multicenter validation cohort: $n=158)$. MGR was computed during the pretreatment period before initiation of therapy with anti-PD-1 antibodies nivolumab or pembrolizumab by measuring the increase of the longest diameter of the largest target lesion. Tumor doubling time served as quality control. Kaplan-Meier analysis and univariable as well as multivariable Cox regression were used to examine the prognostic impact of MGR.

Results Pretreatment MGR $>3.9 \mathrm{~mm} /$ month was associated with impaired OS in the discovery cohort (HR $6.19,95 \% \mathrm{Cl} 2.92$ to $13.10, \mathrm{p}<0.0001$ ), in the confirmation cohort (HR 3.62, 95\% Cl 2.19 to 5.98, $\mathrm{p}<0.0001$ ) and in the independent validation cohort (HR 2.57, 95\% Cl 1.56 to $4.25, p=0.00023)$. Prior lines of systemic treatment did not influence the significance of MGR. Importantly, the prognostic impact of MGR was independent of total tumor burden, diameter of the largest metastasis, number of prior lines of systemic treatment, LDH, as well as liver and brain metastasis (discovery and confirmation cohorts: both $p<0.0001)$. Superiority of MGR compared with these variables was confirmed in the independent multicenter validation cohort (HR 2.92, 95\% Cl 1.62 to 5.26, $p=0.00036$ ).

Conclusions High pretreatment MGR is an independent strong prognostic biomarker associated with unfavorable survival of melanoma patients receiving anti-PD-1 antibodies. Further investigations are warranted to assess the predictive impact of MGR in distinct systemic therapeutic regimens.

\section{BACKGROUND}

Immune checkpoint inhibitors (ICI), namely antibodies against programmed death receptor-1 (PD-1), have considerably improved the outcome of patients with advanced melanoma and are capable to induce long-lasting responses in melanoma patients. ${ }^{12}$ However, primary or acquired resistance against ICI is common and occurs in $50 \%-60 \%$ of the patients. ${ }^{3}$ Therefore, prognostic biomarkers are urgently needed that identify patients who might benefit from anti-PD-1 antibodies more than others.

Clinical experience at our institution suggests that patients displaying extensive tumor burden and fast-growing tumors tend be non-responders to ICI. ${ }^{4}$ This clinical experience is supported by data from Ribas et al indicating that high total tumor burden of more or equal than $102 \mathrm{~mm}$ according to Response Evaluation Criteria in Solid Tumors 
(RECIST) V.1.1 correlates with lower response rates in patients treated with pembrolizumab. ${ }^{5}$ Indirect markers for tumor growth or tumor cell turnover like lactate dehydrogenase (LDH) have been studied extensively in the setting of immunotherapy with anti-PD-1 antibodies and were associated with survival. ${ }^{46-9}$ Thus, the direct investigation of tumor growth as a prognostic marker seems obvious. As early as in the 1960s to 1990s, tumor growth rate (TGR) by means of tumor doubling time (TDT) has been studied in patients with cancer with pulmonary metastases undergoing surgical resection. ${ }^{10} 11$ Only patients with slow-growing pulmonary metastases benefited from surgery and achieved long-term overall survival (OS). ${ }^{10}$ However, only little is known about the impact of TGR in the context of systemic therapy. In 2014, a French study demonstrated the superiority of initial metastatic kinetics compared with $\mathrm{LDH}$ and American joint committee on cancer (AJCC) stage of disease in patients treated with chemotherapy. ${ }^{12} \mathrm{~A}$ recent study reported on fast growing metastases with an intraindividual broad range of TGR being associated with impaired survival in patients treated with BRAF inhibitors (BRAFi). ${ }^{13}$ The group around Hartung et aldetermined pretreatment disease kinetics by measuring every metastasis in each patient. The discovery of hyperprogressive disease in patients receiving ICI brought pretreatment TGR again into a broader focus. However, the results in respect of the prognostic impact of pretreatment TGR were conflicting. ${ }^{14-17}$ Champiat and colleagues even found an inverse correlation of TGR with objective response in a single-center study including 131 patients with 21 distinct cancer entities treated with antibodies directed against PD-1 or programmed cell death 1 ligand 1 (PD-L1). ${ }^{14}$

The aim of this study was to analyze the prognostic impact of pretreatment TGR and total tumor burden on OS in melanoma patients receiving anti-PD-1 antibodies. Moreover, we aimed at developing a feasible method of approximating pretreatment TGR that could replace the ineffective and time-consuming measurement of each metastasis. We hypothesized that high metastatic growth rate (MGR) correlates with inferior survival and lower response rates to anti-PD-1 therapy in patients with metastatic melanoma.

\section{METHODS \\ Patients}

From October 2013 to February 2017, 53 consecutive patients with unresectable melanoma were treated with the anti-PD-1 antibody nivolumab (discovery cohort) and 126 patients with the anti-PD-1 antibody pembrolizumab (confirmation cohort) at the Department of Dermatology, University Hospital Tübingen, Germany and were enrolled retrospectively in this study. A third cohort comprizing 158 melanoma patients treated with either nivolumab or pembrolizumab between February 2013 and September 2019 at 12 distinct clinical sites throughout Austria, Germany and Switzerland (validation cohort) was enrolled as an independent multicentric validation cohort. Online supplemental table 1 summarizes the total number of patients enrolled in the study. Patients were eligible for inclusion if they had a radiographic imaging by CT, MRI, or positron emission tomography-CT (PET$\mathrm{CT})$ at baseline $\left(\mathrm{T}_{0}\right)$ and at least one additional prebaseline staging 28 days or more prior to $\mathrm{T}_{0}\left(\mathrm{~T}_{-1}\right)$. Patient data, clinical variables, and radiologic reports were obtained from electronic patient records, imaging data were evaluated with the study sites' PACS DICOM viewer. The study was carried out in accordance with the Declaration of Helsinki of 1975 and succeeding amendments.

\section{Treatment and response assessment}

Patients received either nivolumab or pembrolizumab in the respective approved dosages or according to the clinical trials' protocols. Clinical response was assessed every 3 months according to RECIST V.1.1. ${ }^{18}$

\section{Determination of MGR}

Radiological measurements were evaluated based on radiological images and/or written findings. For each patient, the largest metastasis at baseline was determined using the longest diameter (D) in axial plane. In case of lymph node metastases, the short axis diameter was used. For the determination of MGR, the absolute metastatic growth in millimeters $(\mathrm{mm})$ was determined as the difference between the diameter of the largest lesion at the baseline staging $\left(\mathrm{D}_{0}\right)$ and at the prebaseline staging $\left(\mathrm{D}_{-1}\right)$. This difference was divided by the number of days elapsed between the prebaseline staging and the baseline staging (t). The resulting value was multiplied with 30.4375 days per month to convert $\mathrm{mm}$ per day to $\mathrm{mm}$ per month $(\mathrm{mm} / \mathrm{month})$. The following equation summarizes this relation:

$$
M G R=\frac{D_{0}-D_{-1}}{t} \times 30.4375 \frac{\text { days }}{\text { month }}
$$

Putatively inactive metastases, for example, curatively irradiated metastases that were constant in size or regressing before anti-PD-1 treatment was commenced, were not considered for MGR determination. In these cases, the next largest metastasis was chosen. However, only a very few lesions qualifying as target lesions had been irradiated before treatment with anti-PD-1 was commenced (discovery cohort: $n=3$, confirmation cohort: $n=1$, validation cohort: $n=2$ ). Neither their inclusion, nor their omission significantly altered the results. Assessment of clinical response and MGR was performed independently in a blinded fashion. Four experienced reference radiologists (BK, CZ, MP and NR) were involved in this work. Throughout the study, including the 12 independent external study sites, the same methods as well as the same standards to assess radiologic data and radiologic reports were used. As a second measure, tumor growth dynamics was determined by means of the TDT. TDT was determined using the same target lesion utilized for MGR calculation. Tumor volume (V) was approximated by $V=\frac{4}{3} \pi R^{3}$, where $\mathrm{R}$, the radius of the sphere, 
is equal to $\mathrm{D} / 2$. The tumor volumes at baseline $\left(\mathrm{V}_{0}\right)$ and at prebaseline $\left(\mathrm{V}_{-1}\right)$ together with the elapsed time in days between these staging examinations $(\mathrm{t})$ were used to calculate TDT using the following equation according to Honda et $a l^{19}$ :

$$
T D T=\frac{t \times \log (2)}{\log \left(\frac{V_{0}}{V_{-1}}\right)}
$$

\section{Statistical analysis}

Response according to RECIST criteria V.1.1, OS defined as the time from starting anti-PD-1 ICI until death due to any cause or end of follow-up, and progression-free survival (PFS) defined as the time from starting antiPD-1 treatment until progression or death due to melanoma or end of follow-up were explored in all patients. OS and PFS were analyzed using Kaplan-Meier estimator and two-sided log-rank test as well as with multivariable Cox regression analysis. HR in univariable analyses were determined using univariable Cox regression analysis. The cut-off points for MGR and TDT were determined based on the data of the discovery cohort using a previously described algorithm that minimizes the resulting p value. ${ }^{20}$ The obtained cut-off values were applied at all survival analyses throughout the study.

Two-sided Mann-Whitney $\mathrm{U}$ test was used to compare MGR according to best overall response. Categorical variables were compared using two-sided Fisher's exact test. Throughout all analyses, $\mathrm{p}<0.05$ were considered statistically significant. All analyses were performed using $\mathrm{R}$ V.4.0.2 and the 'survival' and 'maxstat' packages. ${ }^{21}$

\section{RESULTS \\ Patient characteristics}

Three hundred and thirty-seven patients with unresectable metastatic melanoma were included in this study (53 patients in the discovery cohort, 126 patients in the confirmation cohort and 158 patients in the independent validation cohort). Detailed clinical characteristics are summarized in table 1 . Most patients started immunotherapy at stage M1c (AJCC classification from 2009) disease $(79.2 \%, 78.6 \%$, and $71.5 \%$, respectively) and had visceral metastases other than lung metastases $(73.6 \%, 66.7 \%$, and $58.2 \%$, respectively). Central nervous system (CNS) metastases were present in $28.3 \%$ of the patients in the discovery cohort, in $35.7 \%$ in the confirmation cohort, and in $20.3 \%$ in the validation cohort. Liver metastases were present in $32.1 \%, 27.8 \%$, and 29.7.\%, respectively. Anti-PD-1 immune checkpoint blockade was implemented as first line treatment in $22.6 \%, 38.1 \%$, and $54.4 \%$ of the patients, respectively. Median OS in the three cohorts was 16.7 months $(95 \%$ CI 12.9 to not reached), 23.4 months (95\% CI 16.8 to not reached), and 38.8 months (95\% CI 31.2 to not reached), respectively.
Table 1 Characteristics of the study population

\begin{tabular}{|c|c|c|c|}
\hline & $\begin{array}{l}\text { Discovery } \\
\text { cohort } \\
(n=53) \\
\text { No }(\%)\end{array}$ & $\begin{array}{l}\text { Confirmation } \\
\text { cohort } \\
(n=126) \\
\text { No }(\%)\end{array}$ & $\begin{array}{l}\text { Validation } \\
\text { cohort } \\
(n=158) \\
\text { No (\%) }\end{array}$ \\
\hline \multicolumn{4}{|l|}{ Age (years) } \\
\hline$\leq 60$ & $31(58)$ & $45(35.7)$ & $45(28.5)$ \\
\hline$>60$ & $22(42)$ & $81(64.3)$ & $113(71.5)$ \\
\hline \multicolumn{4}{|l|}{ Gender } \\
\hline Female & $21(40)$ & 49 (38.9) & $53(33.5)$ \\
\hline Male & $32(60)$ & $77(61.1)$ & $105(66.5)$ \\
\hline \multicolumn{4}{|l|}{ BRAF mutational status } \\
\hline Negative & $37(70)$ & $77(61.1)$ & $100(63.3)$ \\
\hline Positive & $16(30)$ & $46(36.5)$ & $57(36.1)$ \\
\hline Unknown & $0(0)$ & $3(2.4)$ & $1(0.6)$ \\
\hline \multicolumn{4}{|l|}{ No of organs involved } \\
\hline 1 & $7(13)$ & $15(11.9)$ & $32(20.3)$ \\
\hline 2 & $15(28)$ & $40(31.7)$ & $35(22.2)$ \\
\hline 3 & $14(26)$ & $26(20.6)$ & $43(27.2)$ \\
\hline 4 & $7(13)$ & $19(15.1)$ & $34(21.5)$ \\
\hline 5 & $7(13)$ & $14(11.1)$ & $6(3.8)$ \\
\hline 6 & $2(4)$ & $7(5.6)$ & $7(4.4)$ \\
\hline$\geq 7$ & $1(2)$ & $5(4.0)$ & $1(0.6)$ \\
\hline \multicolumn{4}{|c|}{ AJCC M stage (AJCC 2009) } \\
\hline Mo & $1(2)$ & $3(2.4)$ & $8(5.1)$ \\
\hline M1a & $2(4)$ & $4(3.2)$ & $10(6.3)$ \\
\hline M1b & $8(15)$ & $20(15.9)$ & $27(17.1)$ \\
\hline M1c & $42(79)$ & 99 (78.6) & $113(71.5)$ \\
\hline \multicolumn{4}{|l|}{ Visceral metastasis } \\
\hline No & $14(26)$ & 42 (33.3) & $66(41.8)$ \\
\hline Yes & $39(74)$ & $84(66.7)$ & $92(58.2)$ \\
\hline \multicolumn{4}{|l|}{ CNS metastasis } \\
\hline No & $38(72)$ & $81(64.3)$ & $126(79.7)$ \\
\hline Yes & $15(28)$ & $45(35.7)$ & $32(20.3)$ \\
\hline \multicolumn{4}{|l|}{ Liver metastasis } \\
\hline No & $36(68)$ & $91(72.2)$ & $111(70.3)$ \\
\hline Yes & $17(32)$ & $35(27.8)$ & $47(29.7)$ \\
\hline \multicolumn{4}{|c|}{ Prior treatment regimens } \\
\hline Anti-CTLA-4 & $36(68)$ & 54 (42.9) & $51(32.3)$ \\
\hline Anti-PD-1 & $0(0)$ & $4(3.2)$ & $11(7.0)$ \\
\hline $\mathrm{BRAFi} \pm \mathrm{MEKi}$ & $10(19)$ & $34(27.0)$ & $29(18.4)$ \\
\hline MEKi & $3(6)$ & $0(0.0)$ & $4(2.5)$ \\
\hline Chemotherapy & $9(17)$ & $28(22.2)$ & $15(9.5)$ \\
\hline Radiotherapy & $29(55)$ & $53(42.1)$ & $40(25.3)$ \\
\hline Adjuvant interferon & $17(32)$ & 49 (38.9) & $31(19.6)$ \\
\hline Other & $1(2)$ & $1(0.8)$ & $1(0.6)$ \\
\hline \multicolumn{4}{|l|}{ Line of treatment } \\
\hline First line & $12(23)$ & $48(38.1)$ & $87(55.1)$ \\
\hline Second line & $24(45)$ & $40(31.7)$ & $39(24.7)$ \\
\hline$\geq$ Third line & $17(32)$ & $38(30.2)$ & $32(20.3)$ \\
\hline
\end{tabular}


Table 1 Continued

$\begin{array}{lll}\begin{array}{l}\text { Discovery } \\ \text { cohort }\end{array} & \begin{array}{l}\text { Confirmation } \\ \text { cohort } \\ (n=53)\end{array} & \begin{array}{l}\text { Validation } \\ \text { cohort } \\ (n=156)\end{array} \\ \text { No }(\%) & \text { No }(\%) & \text { No }(\%)\end{array}$

AJCC 2009 refers to the AJCC staging guideline for melanoma from 2009.

AJCC, American Joint Committee on Cancer; BRAFi, BRAF inhibitors; CNS, central nervous system; CNS, central nervous system; PD-1, programmed death receptor- 1 .

\section{MGR is strongly associated with $\mathrm{OS}$ and predicts response to anti-PD-1 antibodies}

Figure 1 graphically depicts MGR determination and illustrates generic CT image examples of slow-growing and fast-growing metastases. Univariable analysis of OS in the discovery cohort revealed significantly impaired OS in patients with MGR $>3.9 \mathrm{~mm} /$ month compared with the remaining patients (HR 6.19, 95\% CI 2.92 to $13.10, \mathrm{p}<0.0001$ ) (figure $2 \mathrm{~A}$ ). Two-year OS was $8.0 \%$ $(2.1 \%-30.2 \%)$ vs $64.3 \%(48.8 \%-84.7 \%)$. This result was confirmed in the confirmation cohort (HR 3.62, 95\% CI 2.19 to $5.98, \mathrm{p}<0.0001,2$ years OS: $24.9 \%$ (95\% CI $15.2 \%$ to $40.9 \%$ ) vs $62.3 \%$ ( $95 \%$ CI $51.3 \%$ to $75.7 \%$ )) (figure 2B), and in the validation cohort (HR 2.57, 95\% CI 1.56 to $4.25, \mathrm{p}=0.00023$, 2 years OS: $41.4 \%$ (95\% CI $29.4 \%$ to $58.4 \%$ ) vs $80.8 \%$ (95\% CI $72.7 \%$ to $89.8 \%$ )) (figure 2C). TDT less than 37 days was also associated with unfavorable OS (HR 1.83, 95\% CI 1.11 to $3.03, \mathrm{p}=0.018$ ), but its impact was inferior compared with MGR (online supplemental figure 1). Analysis of PFS showed similar results for the three cohorts with MGR being strongly associated with reduced PFS (online supplemental figure 2). Importantly, the results for MGR and OS remained highly significant after exclusion of mucosal and uveal melanomas (online supplemental figure 3 ). In the pooled subgroup of mucosal melanoma, MGR only showed a nonsignificant trend (HR $1.86,95 \%$ CI 0.52 to $6.63, \mathrm{p}=0.34$ ), but in uveal melanoma patients, high MGR significantly correlated with impaired OS (HR 5.87, 95\% CI 1.04 to 32.97, $\mathrm{p}=0.045$ ) (online supplemental figure 4).

Analysis of best objective response according to MGR showed a significant surplus of patients with progressive disease (PD) in the subgroup with MGR exceeding 3.9 $\mathrm{mm} /$ month in the discovery cohort (OR 28.4, 95\% CI 5.9 to $187.5, \mathrm{p}<0.0001$ ), in the confirmation cohort (OR $5.1,95 \%$ CI 2.2 to $12.5, \mathrm{p}<0.0001)$, and in the validation cohort (OR 3.7, 95\% CI 1.7 to 8.1, $\mathrm{p}=0.00036$ ) (table 2). Median MGR was significantly higher in patients reaching PD as best objective response compared with patients with stable disease, partial response or complete response (online supplemental figure 5).

Multivariable Cox regression analysis of OS including MGR, diameter of the largest target lesion, sum of target lesions according to RECIST V.1.1 criteria, liver metastasis, CNS metastasis, $\mathrm{LDH}$, and line of treatment revealed MGR as the only factor being significantly associated with OS in all three cohorts (discovery cohort: HR 9.1, 95\% CI
3.2 to $25.4, \mathrm{p}<0.0001$; confirmation cohort: HR $3.8,95 \%$ CI 2.1 to $6.7, \mathrm{p}<0.0001$; validation cohort: HR $2.9,95 \%$ CI 1.6 to $5.3, \mathrm{p}=0.00036$ ) (table 3 ).

Based on the unexpected minor importance of $\mathrm{LDH}$ in multivariable analysis, the respective impact of $\mathrm{LDH}$ and MGR on OS was assessed in a combined Kaplan-Meier analysis of the pooled cohorts (figure 3). While LDH barely separated the MGR low and MGR high subgroups (MGR low: HR 1.30, 95\% CI 1.02 to $1.65, \mathrm{p}=0.036$; MGR high: HR 1.21, 95\% CI 0.98 to $1.49, \mathrm{p}=0.076$ ), the differences according to MGR was highly significant both in the LDH low (upper limit of normal, $\leq \mathrm{ULN}$ ) and LDH high (>ULN) subgroups (LDH low: HR 3.75, 95\% CI 2.33 to 6.02, $\mathrm{p}<0.0001$; LDH high: HR 2.69, $95 \%$ CI 1.74 to 4.18 , $\mathrm{p}<0.0001)$. These results were underlined by the comparison of LDH low-MGR high with LDH high-MGR low patients (HR 0.51, 95\% CI 0.32 to 0.83 , $\mathrm{p}=0.0061$ ).

To investigate whether MGR was prognostic for OS independently of prior therapies, subgroup analyses comprizing patients with any prior systemic therapy, treatment-naïve patients, patients with prior BRAFi therapy, and patients with prior anti-CTLA-4 therapy were conducted. The prognostic impact of MGR on OS (figure 4) and PFS (online supplemental figure 6) was comparable and highly significant $(\mathrm{p}<0.001$, each) throughout these subgroups. The predictive capacity of MGR also remained high in subgroup analysis comprizing either patients who received prior radiotherapy (HR 3.85, $95 \%$ CI 2.35 to $6.31, \mathrm{p}<0.0001)$ or being radiotherapynaïve (HR 2.84, 95\% CI 1.86 to 4.35, p<0.0001) (online supplemental figure 7 ).

\section{DISCUSSION}

Indirect serum biomarkers of tumor burden and tumor cell turnover like LDH are commonly used for prediction of response and monitoring of the course of disease. ${ }^{56822}$ However, they are prone to failure due to limited specificity and their surrogate nature. ${ }^{23} 24$ Therefore, direct approaches for measuring tumor burden and disease kinetics are needed. In the present study, pretreatment MGR was found to represent a reliable prognostic marker strongly correlating with OS and PFS of melanoma patients treated with anti-PD-1 antibodies. In multivariable analysis, pretreatment MGR was clearly superior to the established prognostic factors tumor burden, LDH, liver metastasis and CNS metastasis.

In the last decade, TGR has been of increasing interest due to limitations of the established criteria to evaluate response to anticancer therapy. Several studies could show that the variation of on-treatment compared with pretreatment TGR is superior to the determination of objective response based on RECIST and that a significant number of patients classified with PD showed decreasing TGR under therapy. ${ }^{152526}$ Moreover, with the introduction of ICI, TGR was discovered as a measure to identify patients showing hyperprogressive disease. ${ }^{14} 16172728$ Interestingly, the results of our study are in sharp contrast 
A Determination of metastatic growth rate

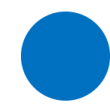

$\mathrm{D}=10 \mathrm{~mm}$ at 100 days before baseline $\left(=D_{-1}\right)$

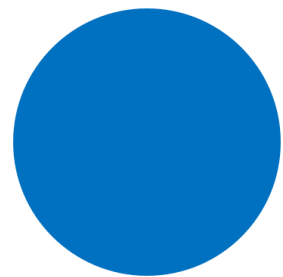

$\mathrm{D}=30 \mathrm{~mm}$ at baseline $\left(=D_{0}\right)$

\section{B Generic examples of slow-growing and fast-growing metastases}

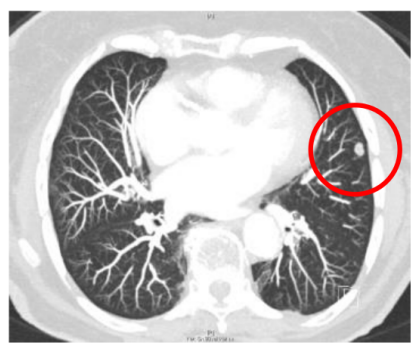

$-727 d$

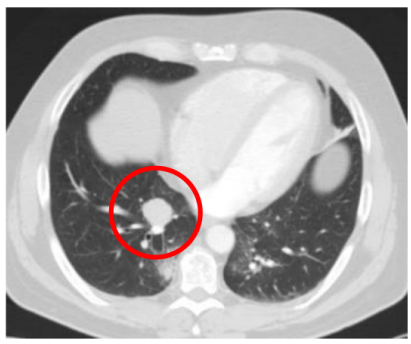

$-91 d$

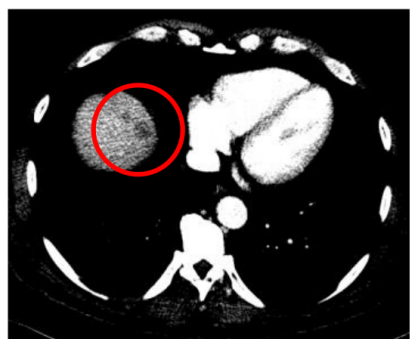

$-87 d$

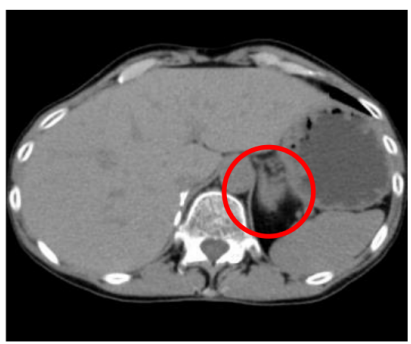

$-76 d$

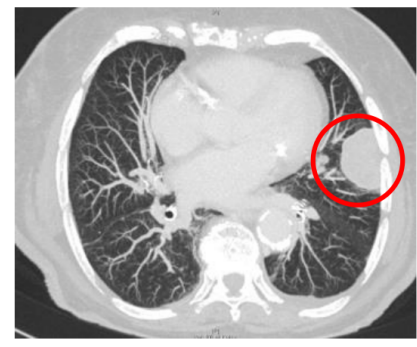

$0 \mathrm{~d}$

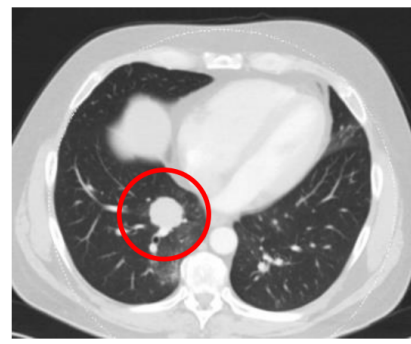

$0 \mathrm{~d}$

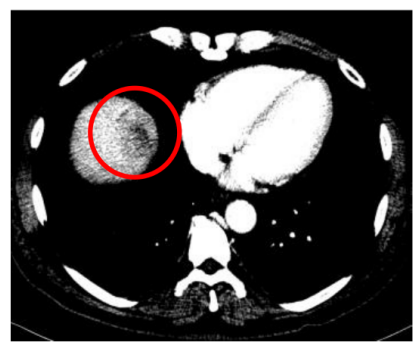

$0 d$

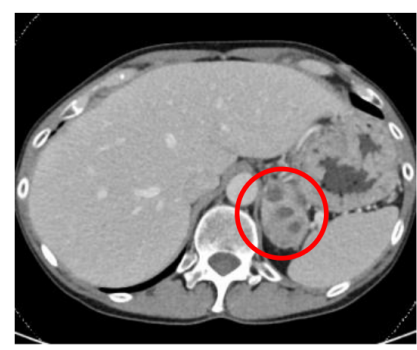

$0 \mathrm{~d}$

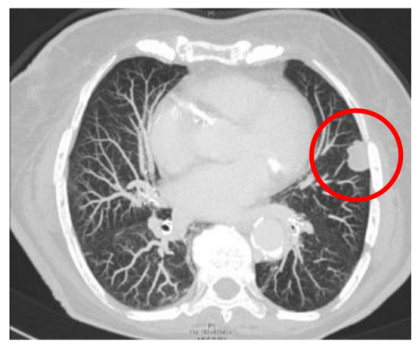

$105 d$

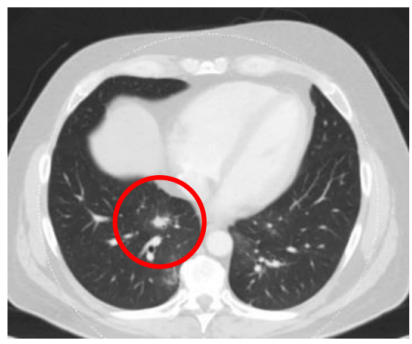

$128 d$

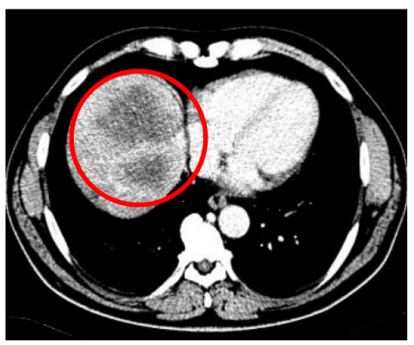

$101 \mathrm{~d}$

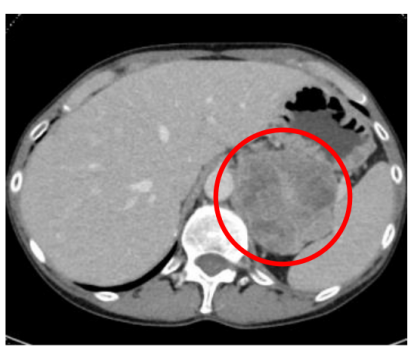

$202 d$

$=1.5 \mathrm{~mm} / \mathrm{month}$

$=0.9 \mathrm{~mm} / \mathrm{month}$

$=7.9 \mathrm{~mm} / \mathrm{month}$

$=8.0 \mathrm{~mm} / \mathrm{month}$

Figure 1 Determination of metastatic growth rate (MGR). (A) Schematic of MGR calculation based on two CT-/MRI-based measurements of metastatic diameters of the largest target lesion. $D_{0}$ is the diameter of the largest target lesion at baseline $\left(T_{0}\right)$, whereas $D_{-1}$ is the diameter of the identical target lesion at the last staging prior to baseline $\left(T_{-1}\right)$. (B) Example CT images of the largest target lesions of two patients with low MGR (upper two rows) and of two distinct patients with high MGR (lower two rows) at the indicated time points. d, days. 


\section{A Discovery Cohort}

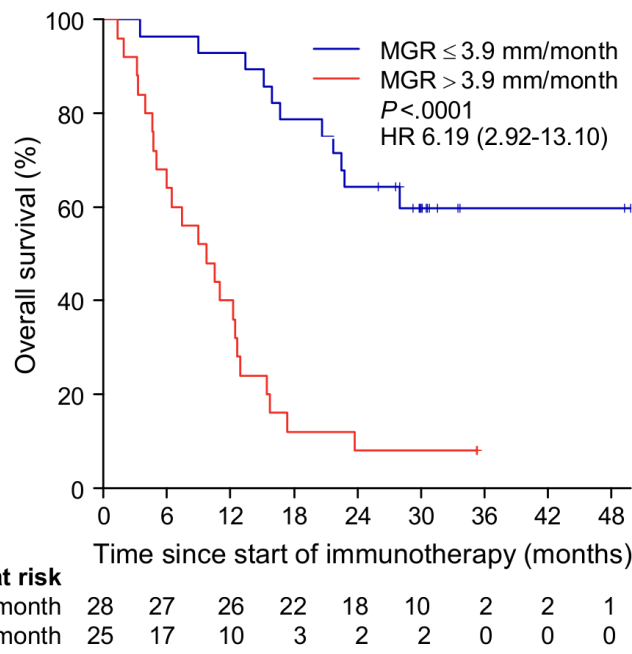

\section{B Confirmation Cohort}

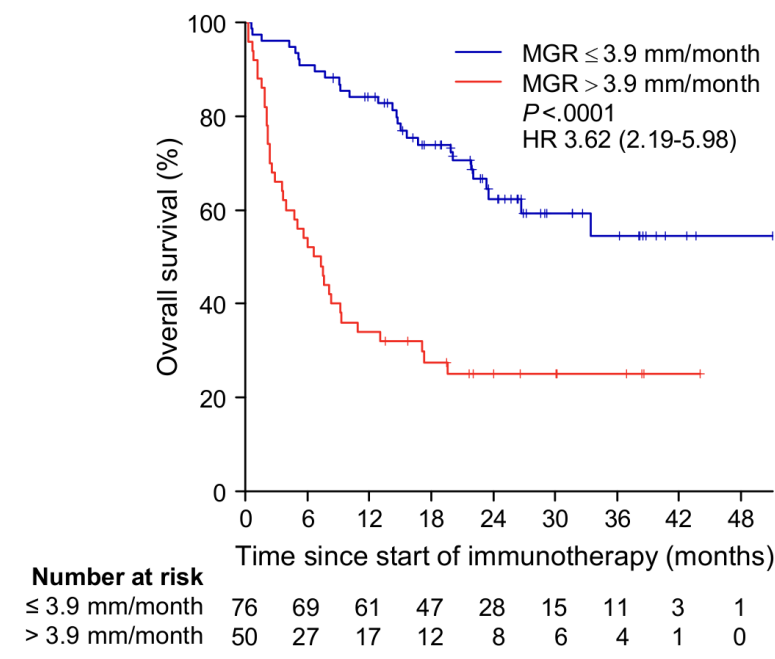

\section{Validation Cohort}

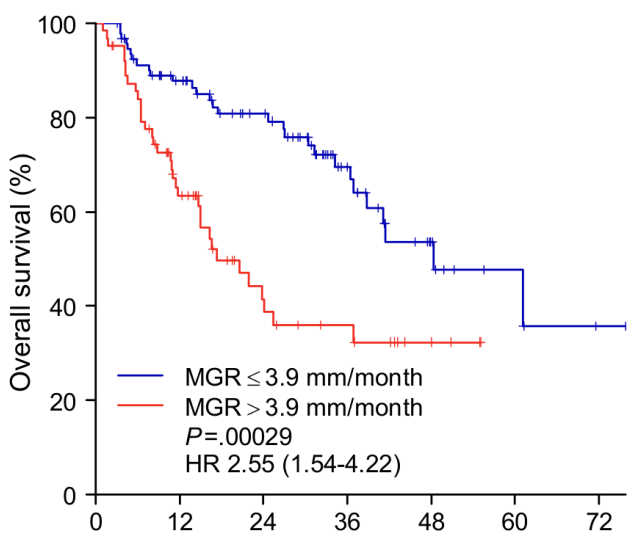

Number at risk

Time since start of immunotherapy (months)

$\begin{array}{llllllll}\leq 3.9 \mathrm{~mm} / \text { month } & 94 & 72 & 51 & 26 & 10 & 4 & 1\end{array}$

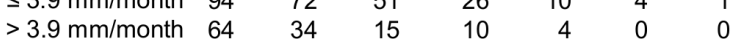

Figure 2 Overall survival according to metastatic growth rate (MGR). Kaplan-Meier curves depicting overall survival according to MGR. PD-1, programmed death receptor-1. to findings of Champiat and colleagues who found an inverse correlation between pretreatment TGR and the percentual change of the sum of RECIST target lesions under therapy with anti-PD-1 or anti-PD-L1 antibodies in a single-cohort study on a heterogeneous set of 131 patients with 21 distinct tumor entities. ${ }^{14}$ The authors drew the conclusion that unlike in targeted therapy, ICI showed improved efficacy in patients exhibiting faster TGR. Importantly, the authors missed to notice the confounding effect of their heterogeneous cohort composed of fast-growing cancers like high-grade glioma (median TDT: 63.4 days) ${ }^{29}$ or melanoma (median TDT: 61 days $)^{30}$ and slow-growing cancers like adenocarcinoma of the lung (median TDT: 258 days) ${ }^{19}$ or clear cell renal cell carcinoma (median TDT: 826 days) ${ }^{31}$ However, antiPD-1 therapy induced response rates and PFS largely differ between these entities (melanoma: objective response rate (ORR) 32\%, median PFS 6.9 months; renal cell carcinoma: ORR 25\%, median PFS 4.6 months; esophagogastric cancer: ORR 12\%, median PFS 1.4 months). ${ }^{22} 3233$

The question whether pretreatment MGR is prognostic in general or specifically predictive for outcome in antiPD-1-treated patients cannot be answered by our study. Although some studies implicated a strong general prognostic impact on survival of cancer patients, ${ }^{10-12}$ the extent of MGR's discriminatory power was unexpectedly impressive in our data. Moreover, besides OS, high MGR was also clearly associated with unfavorable PFS and failure to achieve disease control or an objective response. In multivariable analysis of all three independent cohorts, MGR was most clearly associated with OS compared with the diameter of the largest target lesion, sum of RECIST target lesions, LDH, presence of liver metastasis, and presence of brain metastasis. Superiority of MGR over LDH was confirmed by the combined Kaplan-Meier analysis of both biomarkers that demonstrated the pronounced prognostic impact of MGR compared with LDH.

In comparison to the studies published so far, the main strength of our study is the inclusion of three independent cohorts comprizing a multicenter external validation cohort and 337 patients in total. Therefore, we can conclude with certainty that MGR constitutes a powerful and valuable novel prognostic marker for patients treated with anti-PD-1 antibodies.

Our data suggest that anti-PD-1 antibodies are not capable to inhibit rapidly growing metastases. Recently, Huang et al delineated the ratio of T-cell invigoration to tumor burden as being closely associated with response to anti-PD-1 checkpoint blockade. ${ }^{34}$ Patients with low ratios of Ki67 positive PD-1 positive T-cells to tumor burden exhibited low ORR and impaired survival. In line are recent findings of our group that showed that the interruption of interferon-induced senescence leads to an uncontrolled growth of melanoma metastases. ${ }^{35}$ These results indicate that a disequilibrium between unleashed tumor growth and T-cells leads to fatal outcomes.

In the recent past, several efforts have been made to identify novel prognostic markers in the setting of ICI 
Table 2 Best objective response according to MGR

\begin{tabular}{|c|c|c|c|c|c|c|c|c|c|}
\hline & \multicolumn{3}{|c|}{$\begin{array}{l}\text { Discovery cohort } \\
\text { n (\%) }\end{array}$} & \multicolumn{3}{|c|}{$\begin{array}{l}\text { Confirmation cohort } \\
\mathrm{n}(\%)\end{array}$} & \multicolumn{3}{|c|}{$\begin{array}{l}\text { Validation cohort } \\
\text { n (\%) }\end{array}$} \\
\hline & PD & SD & PR/CR & PD & SD & PR/CR & PD & SD & PR/CR \\
\hline MGR $\leq 3.9 \mathrm{~mm} /$ month & $4(14)$ & $9(32)$ & $15(54)$ & $23(31)$ & $13(17)$ & $39(52)$ & $18(19)$ & $23(24)$ & $53(56)$ \\
\hline \multirow[t]{2}{*}{ MGR $>3.9 \mathrm{~mm} /$ month } & $21(84)$ & $1(4)$ & $3(12)$ & $32(70)$ & $3(7)$ & $11(24)$ & $30(47)$ & $11(17)$ & $23(36)$ \\
\hline & \multicolumn{3}{|c|}{$\begin{array}{l}\left.\mathrm{OR}^{*}: 28.4 \text { (5.9 to } 187.5\right) \\
\mathrm{p}<0.0001\end{array}$} & \multicolumn{3}{|c|}{$\begin{array}{l}\text { OR: } 5.1(2.2 \text { to } 12.5) \\
p<0.0001\end{array}$} & \multicolumn{3}{|c|}{$\begin{array}{l}\text { OR: } 3.7(1.7 \text { to } 8.1) \\
p=0.00036\end{array}$} \\
\hline
\end{tabular}

Best objective response was assessed according to Response Evaluation Criteria in Solid Tumors V.1.1.

${ }^{*} \mathrm{ORs}$ and $\mathrm{p}$ values were determined utilizing two-sided Fisher's exact test comparing PD versus SD/PR/CR.

$\mathrm{CR}$, complete response; MGR, metastatic growth rate; PD, progressive disease; PR, partial response; SD, stable disease.

with PD-1 antibodies. Most promising, but also controversial is the utilization of PD-L1 status as a predictor of response. ${ }^{36}$ Although several studies have highlighted the predictive impact of PD-L1 expression in tumor tissue, several limitations deserve cautious appraisal of this molecular biomarker. ${ }^{2}{ }^{37-40}$ A major disadvantage of PD-L1 status concerns the prerequisite of surgically accessible metastases and its high effort. Moreover, technical issues like PD-L1 expression heterogeneity within the microenvironment hampers the evaluation of immunohistochemistry. ${ }^{36}$

In previous studies, TGR was either computed based on all 1-5 RECIST target lesions, 1-10 iRECIST target lesions, or by measuring the total number of each measurable metastasis. ${ }^{12-17}$ 25-27 The approach used by GaudyMarqueste et al as well as Hartung et al implicated the

Table 3 Multivariable Cox regression analysis of overall survival

\begin{tabular}{|c|c|c|c|c|c|c|}
\hline & \multicolumn{2}{|c|}{$\begin{array}{l}\text { Discovery cohort } \\
(n=53)\end{array}$} & \multicolumn{2}{|c|}{$\begin{array}{l}\text { Confirmation cohort } \\
(n=126)\end{array}$} & \multicolumn{2}{|c|}{$\begin{array}{l}\text { Validation cohort } \\
(n=158)\end{array}$} \\
\hline & HR (95\% Cl) & $P$ value & HR (95\% Cl) & $P$ value & HR (95\% Cl) & $P$ value \\
\hline \multicolumn{7}{|l|}{ MGR } \\
\hline$\leq 3.9 \mathrm{~mm} / \mathrm{month}$ & 1 & & 1 & & 1 & \\
\hline$>3.9 \mathrm{~mm} /$ month & 9.1 (3.2 to 25.4 ) & $<0.0001$ & 3.8 (2.1 to 6.7$)$ & $<0.0001$ & 2.9 (1.6 to 5.3$)$ & 0.00036 \\
\hline \multicolumn{7}{|c|}{ Diameter of largest TL } \\
\hline$\leq$ Median & 1 & & 1 & & 1 & \\
\hline >Median & 0.3 (0.1 to 0.9 ) & 0.022 & 1.3 (0.6 to 3.0$)$ & 0.47 & 0.7 (0.3 to 1.5$)$ & 0.34 \\
\hline \multicolumn{7}{|l|}{ Sum of RECIST TLs } \\
\hline$\leq$ Median & 1 & & 1 & & 1 & \\
\hline$>$ Median & 3.1 (1.0 to 9.6 ) & 0.046 & 0.6 (0.3 to 1.3 ) & 0.16 & 1.3 (0.6 to 2.7 ) & 0.45 \\
\hline \multicolumn{7}{|l|}{ Liver metastasis } \\
\hline No & 1 & & 1 & & 1 & \\
\hline Yes & 2.4 (0.9 to 6.5$)$ & 0.081 & 2.0 (1.1 to 3.4$)$ & 0.016 & 2.0 (1.2 to 3.3$)$ & 0.0088 \\
\hline \multicolumn{7}{|l|}{ CNS metastasis } \\
\hline No & 1 & & 1 & & 1 & \\
\hline Yes & 2.9 (1.1 to 8.2$)$ & 0.039 & 2.2 (1.3 to 3.8$)$ & 0.0058 & 1.3 (0.7 to 2.4$)$ & 0.40 \\
\hline \multicolumn{7}{|l|}{$\mathrm{LDH}$} \\
\hline$\leq \mathrm{ULN}$ & 1 & & 1 & & 1 & \\
\hline$>$ ULN & $1.6(0.7$ to 3.8$)$ & 0.28 & 1.7 (0.9 to 3.1$)$ & 0.081 & 1.5 (0.9 to 2.5$)$ & 0.16 \\
\hline \multicolumn{7}{|l|}{ Line of treatment } \\
\hline First line & 1 & & 1 & & 1 & \\
\hline Second line & 0.8 (0.3 to 2.4$)$ & 0.73 & 0.7 (0.4 to 1.3$)$ & 0.28 & 2.0 (1.1 to 3.9$)$ & 0.033 \\
\hline$\geq$ Third line & 0.9 (0.2 to 3.1$)$ & 0.84 & $1.0(0.5$ to 1.9$)$ & 0.96 & 2.3 (1.2 to 4.3$)$ & 0.012 \\
\hline
\end{tabular}

CNS, central nervous system; LDH, lactate dehydrogenase; MGR, metastatic growth rate; RECIST, response evaluation criteria in solid tumors version 1; ULN, upper limit of normal. 


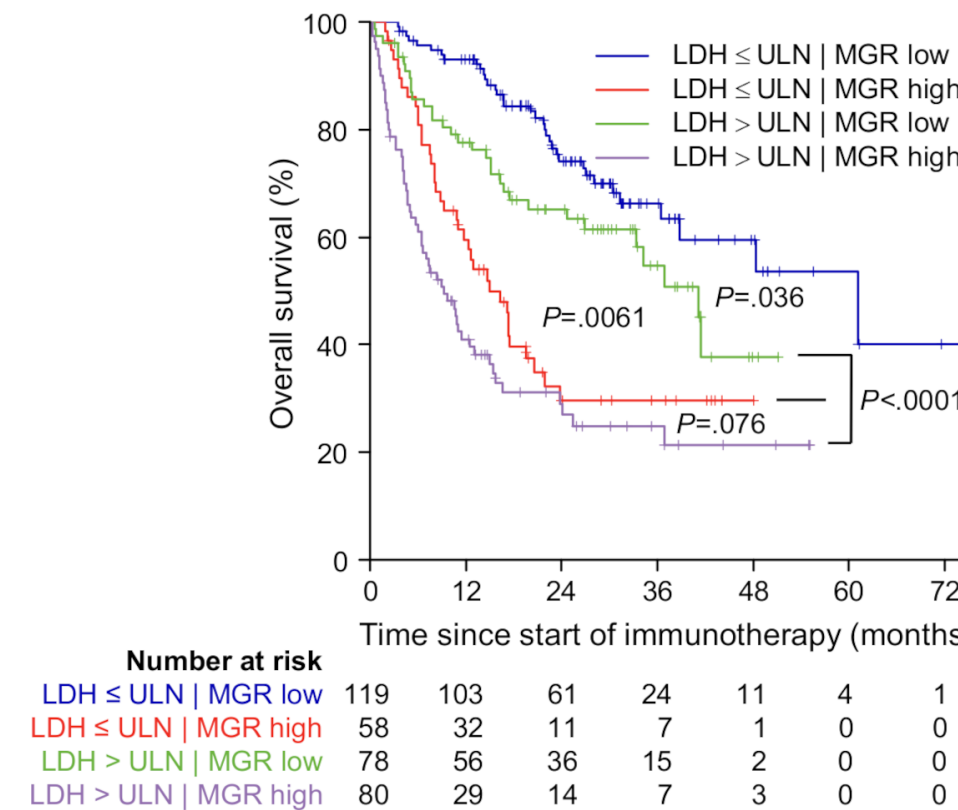

Figure 3 Overall survival according to lactate dehydrogenase (LDH) and metastatic growth rate (MGR) Kaplan-Meier curves depicting overall survival in the pooled entire cohort according to LDH below or above upper limit of normal (ULN) and pretreatment MGR below or above $3.9 \mathrm{~mm} /$ month.

measurement of all metastases with a diameter of at least $10 \mathrm{~mm}$ in three dimensions. ${ }^{12}{ }^{13}$ Especially in patients harboring hundreds of metastases, this approach is very time-consuming and limits sample size. ${ }^{13}$ Data derived from chest roentgenograms had shown little intraindividual variation in TDT of multiple metastatic lesions suggesting that measuring only one target lesion could be sufficient to reflect disease kinetics. ${ }^{41}{ }^{42}$ This justifies our rationale for measuring only one target lesion which seems suitable to reflect tumor growth dynamics. Different models of tumor growth have been developed, comprizing exponential, exponential-linear, Gompertz function, or logistic growth, and there is a long-lasting discussion on what is the best to describe this dynamic process. ${ }^{43-45}$ To take the classical exponential model into account, our study includes the TDT used by many authors. ${ }^{19434647}$ In addition, we introduce a novel approach that, like the logistic or Gompertz models, considers the declining growth rate of larger metastases when angiogenesis, nutrient and oxygen depletion, as well as tumor cell necrosis increasingly play a role. ${ }^{44} 45$ While TDT is constant at 30 days per doubling for two lesions that increase from 10 to $20 \mathrm{~mm}$ (diameter) within 90 davs, and from 40 to $80 \mathrm{~mm}$ within 90 days, respectively, MGR reflects this more dramatic absolute growth of the larger lesion with calculated growth rates of $3.4 \mathrm{~mm} /$ month and $13.5 \mathrm{~mm} /$ month, respectively. Murphy et al presented in detail with impressive examples that all models of tumor growth encounter their limits under certain conditions. ${ }^{48}$ Despite these considerations, the herewith introduced MGR, although not claiming to represent a theoretical mathematical model of tumor growth, was capable to discriminate the patients more efficiently than TDT.
We are aware of several limitations of our study. The retrospective design makes it susceptible for a patient selection bias. However, we included all consecutive patients receiving nivolumab or pembrolizumab who had at least one prebaseline staging at our center. Moreover, a third independent cohort of patients enrolled at 12 participating centers and assessed by 12 independent and experienced dermato-oncologists and radiologists confirmed the results observed in the two monocentric cohorts. Thereby, site-specific treatment procedures and patient selection bias could be minimized. To the best of our knowledge, this is by far the largest set of patients analyzed concerning pretreatment tumor growth kinetics.

Another putative limitation constitutes the impact of prior treatment regimens on MGR. In the discovery cohort 36 of the 53 patients had been previously treated with ipilimumab. It seems suggestive that patients showing stable or even slightly decreasing target lesions on treatment with ipilimumab might benefit from an anti-PD-1 antibody or tend to benefit from any ICI. However, MGR remained a strong prognostic factor in subgroup analysis of patients with any prior systemic therapy, prior BRAFi, prior ipilimumab, and treatment-naïve patients.

The probably most important limitation of the concept of pretreatment tumor growth dynamics as baseline prognostic factor is the prerequisite of at least one prebaseline staging. In a personal statement by Jean Jacques Grob, Georgina Long, Dirk Schadendorf and Keith Flaherty published in 2015, the expert authors had discussed the option of postponing the start of therapy to achieve this premise. ${ }^{11} 1231$ However, ethical reservations should be discussed carefully when deciding about treatment delays owed to diagnostic procedures. Given a median MGR 


\section{A Prior systemic therapies}

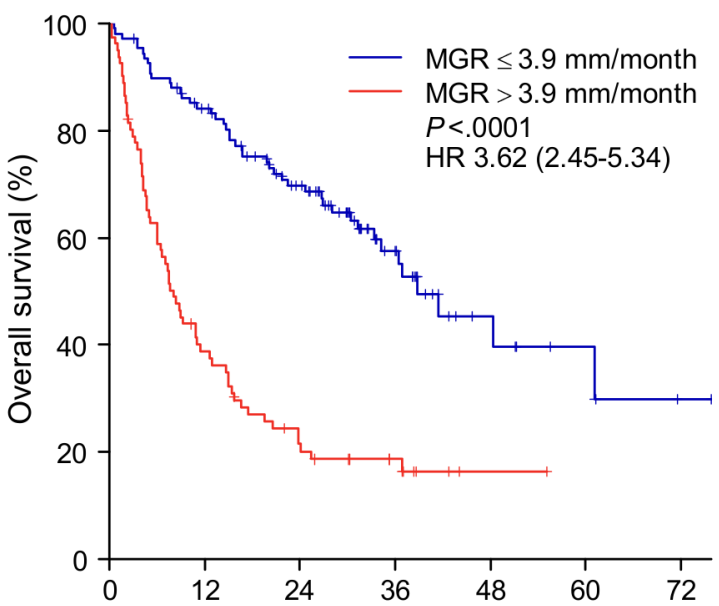

Number at risk

$\begin{array}{llllllll}\leq 3.9 \mathrm{~mm} / \text { month } & 109 & 87 & 61 & 26 & 8 & 4 & 1\end{array}$

$>3.9 \mathrm{~mm} / \mathrm{month} \quad 81$

$\begin{array}{rrrrrr}87 & 61 & 26 & 8 & 4 & 1 \\ 30 & 15 & 8 & 1 & 0 & 0\end{array}$

\section{Prior BRAFi therapy}

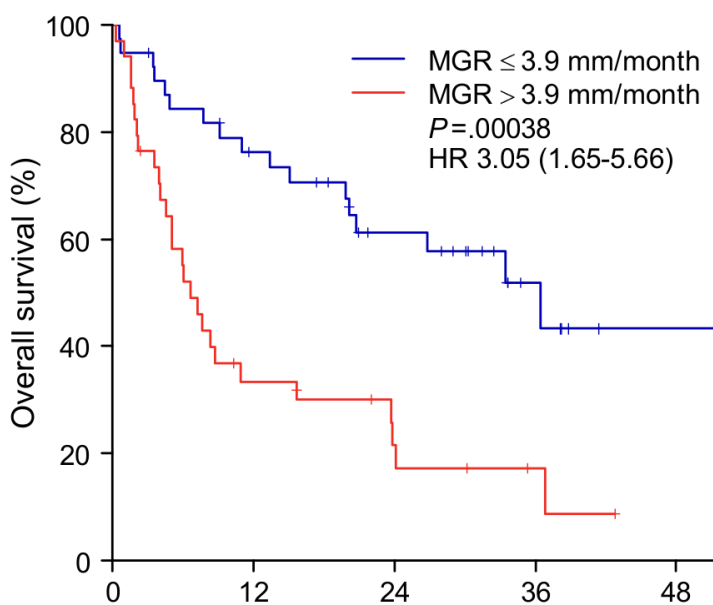

Number at risk

$\leq 3.9 \mathrm{~mm} /$ month 39

$>3.9 \mathrm{~mm} /$ month 34

Time since start of immunotherapy (months)

$\begin{array}{rrrr}27 & 17 & 6 & 1\end{array}$ according to pretreatment MGR in $(A)$ patients with prior systemic therapy/therapies, $(B)$ treatment-naïve patients, $(C)$ patients with prior BRAFi therapy and (D) patients with prior anti-CTLA-4 therapy. BRAFi, BRAF inhibitor; MGR, metastatic growth rate.

of approximately $3 \mathrm{~mm} /$ month and a median doubling time of 41 days, consecutive staging examinations should be separated by at least 1 month to account for measuring inaccuracy. This recommendation for a minimum and an ideal time interval between the two successive measures of target lesions is in accordance with previous suggestions. ${ }^{12}{ }^{49}$ In our validation cohort, the median interval between the pretreatment staging examinations was 86 days (IQR: 61-116 days) indicating a low risk for measuring inaccuracy.

Apart from intentional treatment delays, there can be several other reasons that normally lead to consecutive

\section{B Treatment-naïve patients}

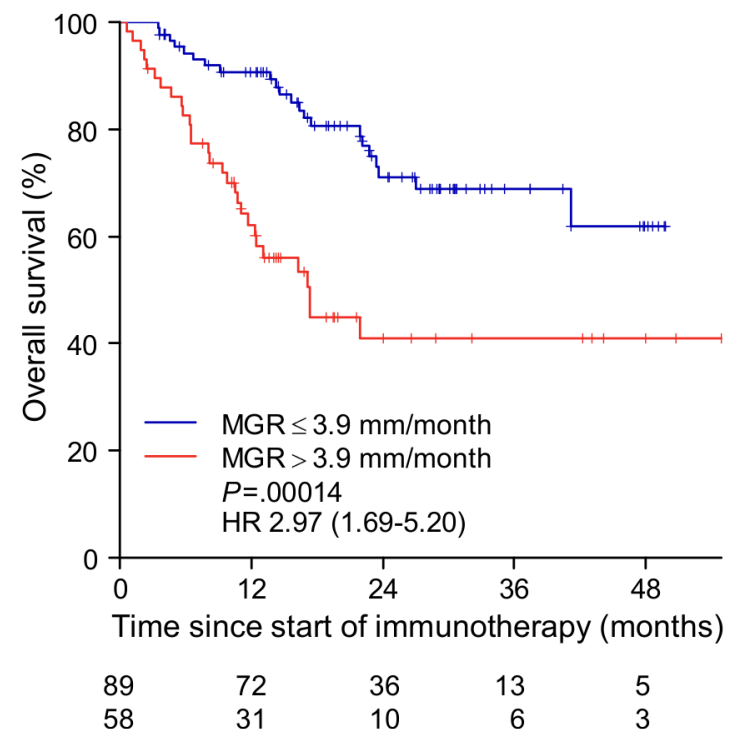

D Prior anti-CTLA-4 therapy

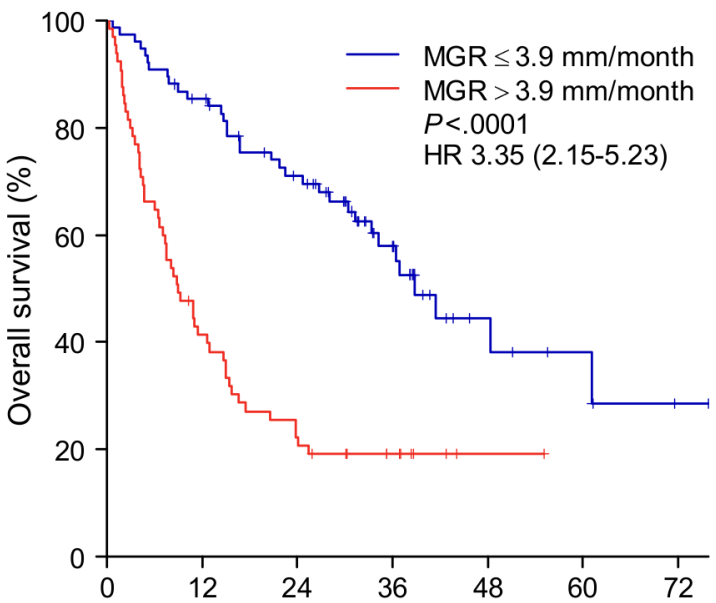

Time since start of immunotherapy (months)

$\begin{array}{rrrrrrr}76 & 63 & 47 & 24 & 7 & 4 & 1 \\ 65 & 26 & 14 & 7 & 1 & 0 & 0\end{array}$




\section{CONCLUSIONS}

In conclusion, high pretreatment TGR is associated with unfavorable outcome and non-response in advanced melanoma patients treated with anti-PD-1 antibodies. With MGR, we propose a novel measure of tumor growth kinetics that independently predicts survival, superiorly compared with total tumor burden, $\mathrm{LDH}$, site of metastasis and other known factors. As a time-efficient method, determination of MGR can be easily implemented in routine clinical settings and should be explicitly considered prior to therapeutic decisions. Investigation of the predictive impact of MGR in distinct therapeutic regimens like PD-1 blockade, combined ICI with anti-CTLA-4 and anti-PD-1, and small molecule inhibitors targeting the MAPK pathway, is warranted.

\section{Author affiliations}

${ }^{1}$ Department of Dermatology, University Hospital Tuebingen, Tuebingen, Germany

${ }^{2}$ Department of Dermatology and Allergology, Kantonsspital St Gallen, Sankt Gallen, Switzerland

${ }^{3}$ Department of Dermatology, University Hospital Carl Gustav Carus, Dresden, Germany

${ }^{4}$ National Center for Tumor Diseases (NCT), Partner Site Dresden, Dresden, Germany ${ }^{5}$ Department of Dermatology, Venereology and Allergology, Medical University of Innsbruck, Innsbruck, Austria

${ }^{6}$ Department of Dermatology, University Medical Center Mainz, Mainz, Germany ${ }^{7}$ Department of Diagnostic and Interventional Radiology, University Medical Centre of the Johannes Gutenberg University Mainz, Mainz, Germany

${ }^{8}$ Department of Dermatology, Allergy, and Venereology, University of Lubeck, Lubeck, Germany

${ }^{9}$ Department of Dermatology, University Hospital Zurich, Zurich, Switzerland ${ }^{10}$ Institute of Diagnostic and Interventional Radiology, University Hospital Zurich, Zurich, Switzerland

${ }^{11}$ Department of Dermatology and Allergology, Paracelsus Medical University Salzburg, Salzburg, Austria

${ }^{12}$ Institute of Radiology, Paracelsus Medical University Salzburg, Salzburg, Austria

${ }^{13}$ Department of Dermatology, Venereology and Allergology, University Hospital Leipzig, Leipzig, Germany

${ }^{14}$ Otto Loewi Research Center, Pharmacology Section, Medical University of Graz, Graz, Austria

${ }^{15}$ Department of Dermatology, Medical University of Graz, Graz, Austria

${ }^{16}$ Department of Dermatology, Ludwig Maximilians University of Munich, Munich, Germany

${ }^{17}$ Department of Dermatology, Johannes Kepler University Linz, Linz, Austria

${ }^{18}$ Department of Trauma Surgery, Technical University of Munich, Munich, Germany

${ }^{19}$ Department for Diagnostic and Interventional Radiology, Eberhard Karls University Tübingen, Tubingen, Germany

${ }^{20}$ Department for Diagnostic and Interventional Radiology, Rems-Murr-Kliniken gGmbH, Winnenden, Germany

${ }^{21}$ Department of Dermatology, Friedrich-Alexander University Erlangen-Nuremberg, Erlangen, Germany

${ }^{22}$ Department of Dermatology and Venereology, University Medical Center HamburgEppendorf, Hamburg, Germany

${ }^{23}$ Department of Oncology and Hematology, Kantonsspital St Gallen, Sankt Gallen, Switzerland

\section{Presented at}

The preliminary results of this article were presented as an oral presentation in the best of session at the 28th German Skin Cancer Congress 2018; September 13 2018; Stuttgart, Germany, as a poster at the ADF 46th Annual Meeting 2019; March 15 2019; Munich, Germany, and was awarded with the Swiss Skin Cancer Award 2020 at the 102nd SSDV (Swiss Society for Dermatology and Venereology) Congress 2020; September 17 2020; Zurich, Switzerland.

Acknowledgements thank the whole team of the melanoma and skin cancer units and all collaborators for their passionate patient care and support in data collection.
NBW is supported by a joint program of Swiss Academy of Medical Sciences (SAMS/SAMW) and Gottfried and Julia Bangerter-Rhyner Foundation.

Contributors NBW was the senior author of the study, developed the concept, designed the study, collected data, contributed statistical review, interpreted the results and drafted the manuscript together with TE. MML, KK, LR, FA, MD, NR, CE, $\mathrm{RS}, \mathrm{CZ}, \mathrm{RL}, \mathrm{MP}, \mathrm{PG}, \mathrm{GR}, \mathrm{SHS}, \mathrm{SK}, \mathrm{AO}, \mathrm{BK}$ and CGe contributed to the acquisition and analysis of data. AF, UL, BW, MG, WH, CB, ER, MZ, JM, PT, CL, VAN, CGe, FM, SD, AC, LF, MR, CGa and TE provided clinical data. NBW, NR, CZ, MP, BK and CGe performed radiological evaluation. All authors took part in interpretation of data. All authors contributed to critical revision of the manuscript for important intellectual content. All authors read and approved the final manuscript.

Funding The authors have not declared a specific grant for this research from any funding agency in the public, commercial or not-for-profit sectors.

Competing interests NBW reports a consulting/advisory role for Sanofi and has received travel support from AbbVie and Amgen outside the submitted work. RL has received research funding from Bristol-Myers Squibb, Pierre Fabre and Roche, honoraria and travel support from Merck Sharp \& Dohme and travel support from Amgen. A0 is a consultant/advisory board member for MSD and Bristol-Myers Squibb. AF has received speaker's honoraria from Bristol-Myers Squibb, MSD, Roche, and Novartis, is advisory board member for Roche and Novartis and received travel support from Roche, Novartis, and Bristol-Myers Squibb. UL is a consultant/advisory board member for and has received honoraria from MSD, Novartis, and Roche. MG is an advisory board member of Novartis and has received grants from Novartis outside the submitted work. CB has received speaker's honoraria and consultancy fees from Amgen, Bristol-Myers Squibb, Merck, MSD, Novartis, Pierre Fabre, Regeneron, Roche, and Sanofi-Aventis outside the submitted work, and has received travel support from Bristol-Myers Squibb. ER has received honoraria from and has a consultancy or advisory role for Amgen, Bayer, Bristol-Myers Squibb, MSD, Merck, Novartis, Pierre Fabre, Roche and Sanofi, reports speakers' bureau for Amgen, Bristol-Myers Squibb, MSD, Merck, Novartis, Pierre Fabre and Sanofi, received research funding (at the institution) from Amgen, Bristol-Myers Squibb, MSD, Novartis, Pierre Fabre and Roche, is a member of the research funding steering committee of Novartis, and received travel accommodations and/or expenses from Amgen, Bristol-Myers Squibb, MSD, Merck, Novartis, Pierre Fabre, Roche and Sanofi. JM has intermittent project focused consultant or advisory relationships with Merck/Pfizer, Merck Sharp \& Dohme, Amgen, Novartis, Bristol-Myers Squibb and Pierre Fabre, and has received travel support from Ultrasun, L'Oreal, Merck Sharp \& Dohme, Bristol-Myers Squibb and Pierre Fabre outside of the submitted work. PT has received honoraria and/ or consultancy fees from Bristol-Myers Squibb, Curevac, Merck, MSD, Novartis, Pierre Fabre, Roche, and Sanofi outside the submitted work, and has received travel support from Bristol-Myers Squibb and Pierre-Fabre. CL is a consultant/advisory board member for and has received advisory board fees, speaker's fees and/or travel reimbursements from Roche, Pierre Fabre, Novartis, Bristol-Myers Squibb, Merck, MSD, Biontech, Almiral Hermal, Kyowa Kirin, Sun Pharma and Sanofi. VAN has received speaker's honoraria and consultancy fees from Amgen, Bristol-Myers Squibb, GlaxoSmithKline, Merck Sharp \& Dohme, Novartis, Roche, and Takeda outside of the submitted work. CGe has received personal speaker/advisory board fees from Amgen, Beiersdorf, BMS, MSD, Novartis, Pierre Fabre, Roche, Sun Pharma and Sanofi Genzyme. FM has received travel support or/and speaker's fees or/and advisor's honoraria by Novartis, Roche, BMS, MSD and Pierre Fabre, and research funding from Novartis and Roche. SD has received travel support from MSD. AC has received consultancy fees from AbbVie, BMS, Almirall, Amgen, Eli Lilly, Galderma, Janssen, Kyowa Kirin, Leo, Novartis, and Sanofi. LF reports grants from the Swiss National Science Foundation, Swiss Cancer League, Hookipa Pharma and Novartis Foundation as well as an advisory role for Novartis and Bristol-Myers Squibb. CGa has received grants and personal fees from Bristol-Myers Squibb, Roche, Novartis and personal fees from Amgen, MSD and Philogen outside the submitted work. TE is a consultant/advisory board member for Philogen, Bristol-Myers Squibb, Novartis, Roche and Sanofi. No other disclosures were reported.

\section{Patient consent for publication Not required.}

Ethics approval Approval to conduct this study was obtained from the local ethics committee of the Medical Faculty of University Tübingen (project No. 436/2017B02) and from the respective ethics committees of all participating centers. The study was carried out in accordance with the Declaration of Helsinki of 1975 and succeeding amendments.

Provenance and peer review Not commissioned; externally peer reviewed.

Data availability statement Data are available on reasonable request. All data relevant to the study are included in the article or uploaded as online supplemental 
information. Data are available on reasonable request from the corresponding author, NikolausBenjamin.Wagner@kssg.ch.

Supplemental material This content has been supplied by the author(s). It has not been vetted by BMJ Publishing Group Limited (BMJ) and may not have been peer-reviewed. Any opinions or recommendations discussed are solely those of the author(s) and are not endorsed by BMJ. BMJ disclaims all liability and responsibility arising from any reliance placed on the content. Where the content includes any translated material, BMJ does not warrant the accuracy and reliability of the translations (including but not limited to local regulations, clinical guidelines, terminology, drug names and drug dosages), and is not responsible for any error and/or omissions arising from translation and adaptation or otherwise.

Open access This is an open access article distributed in accordance with the Creative Commons Attribution Non Commercial (CC BY-NC 4.0) license, which permits others to distribute, remix, adapt, build upon this work non-commercially, and license their derivative works on different terms, provided the original work is properly cited, appropriate credit is given, any changes made indicated, and the use is non-commercial. See http://creativecommons.org/licenses/by-nc/4.0/.

\section{ORCID iDs}

Nikolaus B Wagner http://orcid.org/0000-0003-4708-2886

Andrea Forschner http://orcid.org/0000-0002-6185-4945

Patrick Terheyden http://orcid.org/0000-0002-5894-1677

Christoffer Gebhardt http://orcid.org/0000-0001-7090-9584

\section{REFERENCES}

1 Robert C, Schachter J, Long GV, et al. Pembrolizumab versus ipilimumab in advanced melanoma. N Engl J Med 2015;372:2521-32.

2 Weber JS, D'Angelo SP, Minor D, et al. Nivolumab versus chemotherapy in patients with advanced melanoma who progressed after anti-CTLA-4 treatment (CheckMate 037): a randomised, controlled, open-label, phase 3 trial. Lancet Oncol 2015;16:375-84.

3 Zou W, Wolchok JD, Chen L. PD-L1 (B7-H1) and PD-1 pathway blockade for cancer therapy: mechanisms, response biomarkers, and combinations. Sci Transl Med 2016;8:328rv4.

4 Weide B, Martens A, Hassel JC, et al. Baseline biomarkers for outcome of melanoma patients treated with pembrolizumab. Clin Cancer Res 2016;22:5487-96.

5 Ribas A, Hamid O, Daud A, et al. Association of pembrolizumab with tumor response and survival among patients with advanced melanoma. JAMA 2016;315:1600-9.

6 Diem S, Kasenda B, Spain L, et al. Serum lactate dehydrogenase as an early marker for outcome in patients treated with anti-PD-1 therapy in metastatic melanoma. Br J Cancer 2016;114:256-61.

7 Callahan MK, Kluger H, Postow MA, et al. Nivolumab plus ipilimumab in patients with advanced melanoma: updated survival, response, and safety data in a phase I dose-escalation study. $J$ Clin Oncol 2018;36:391-8.

8 Nosrati A, Tsai KK, Goldinger SM, et al. Evaluation of clinicopathological factors in PD-1 response: derivation and validation of a prediction scale for response to PD-1 monotherapy. $\mathrm{Br}$ $J$ Cancer 2017;116:1141-7.

9 Wagner NB, Forschner A, Leiter U, et al. S100B and LDH as early prognostic markers for response and overall survival in melanoma patients treated with anti-PD-1 or combined anti-PD-1 plus antiCTLA-4 antibodies. Br J Cancer 2018;119:339-46.

10 Morton DL, Joseph WL, Ketcham AS, et al. Surgical resection and adjunctive immunotherapy for selected patients with multiple pulmonary metastases. Ann Surg 1973;178:360-6.

11 Ollila DW, Stern SL, Morton DL. Tumor doubling time: a selection factor for pulmonary resection of metastatic melanoma. J Surg Oncol 1998;69:206-11.

12 Gaudy-Marqueste C, Archier E, Grob A, et al. Initial metastatic kinetics is the best prognostic indicator in stage IV metastatic melanoma. Eur J Cancer 2014;50:1120-4.

13 Hartung N, Huynh CT-K, Gaudy-Marqueste C, et al. Study of metastatic kinetics in metastatic melanoma treated with B-RAF inhibitors: introducing mathematical modelling of kinetics into the therapeutic decision. PLoS One 2017;12:e0176080.

14 Champiat S, Dercle L, Ammari S, et al. Hyperprogressive disease is a new pattern of progression in cancer patients treated by anti-PD-1/ PD-L1. Clin Cancer Res 2017;23:1920-8.

15 Gomez-Roca C, Koscielny S, Ribrag V, et al. Tumour growth rates and RECIST criteria in early drug development. Eur $J$ Cancer 2011;47:2512-6.
16 Ferrara R, Mezquita L, Texier M, et al. Hyperprogressive disease in patients with advanced non-small cell lung cancer treated with PD-1/ PD-L1 inhibitors or with single-agent chemotherapy. JAMA Oncol 2018;4:1543-52.

17 Matos I, Martin-Liberal J, García-Ruiz A, et al. Capturing Hyperprogressive disease with Immune-Checkpoint inhibitors using RECIST 1.1 criteria. Clin Cancer Res 2020;26:1846-55.

18 Eisenhauer EA, Therasse P, Bogaerts J, et al. New response evaluation criteria in solid tumours: revised RECIST guideline (version 1.1). Eur J Cancer 2009;45:228-47.

19 Honda O, Johkoh T, Sekiguchi J, et al. Doubling time of lung cancer determined using three-dimensional volumetric software: comparison of squamous cell carcinoma and adenocarcinoma. Lung Cancer 2009;66:211-7.

20 Hothorn T, Lausen B. On the exact distribution of maximally selected rank statistics. Comput Stat Data Anal 2003;43:121-37.

21 R Core Team. R: a language and environment for statistical computing. Vienna, Austria R Foundation for Statistical Computing; 2017.

22 Wolchok JD, Chiarion-Sileni V, Gonzalez R, et al. Overall survival with combined nivolumab and ipilimumab in advanced melanoma. $N$ Engl $J$ Med 2017;377:1345-56.

23 Balch CM, Buzaid AC, Soong SJ, et al. Final version of the American joint Committee on cancer staging system for cutaneous melanoma. J Clin Oncol 2001;19:3635-48.

24 Palmer SR, Erickson LA, Ichetovkin I, et al. Circulating serologic and molecular biomarkers in malignant melanoma. Mayo Clin Proc 2011;86:981-90.

25 Le Tourneau C, Servois V, Diéras V, et al. Tumour growth kinetics assessment: added value to RECIST in cancer patients treated with molecularly targeted agents. Br J Cancer 2012;106:854-7.

26 Ferté C, Fernandez M, Hollebecque A, et al. Tumor growth rate is an early indicator of antitumor drug activity in phase I clinical trials. Clin Cancer Res 2014;20:246-52.

27 Saâda-Bouzid E, Defaucheux C, Karabajakian A, et al. Hyperprogression during anti-PD-1/PD-L1 therapy in patients with recurrent and/or metastatic head and neck squamous cell carcinoma. Ann Oncol 2017;28:1605-11.

28 Castello A, Rossi S, Mazziotti E, et al. Hyperprogressive Disease in Patients with Non-Small Cell Lung Cancer Treated with Checkpoint Inhibitors: The Role of ${ }^{18}$ F-FDG PET/CT. J Nucl Med 2020;61:821-6.

29 Fan Z, Liu Y, Li S, et al. Association of tumor growth rates with molecular biomarker status: a longitudinal study of high-grade glioma. Aging 2020;12:7908-26.

30 Lee JH, Gulec SA, Kyshtoobayeva A, et al. Biological factors, tumor growth kinetics, and survival after metastasectomy for pulmonary melanoma. Ann Surg Oncol 2009;16:2834-9.

31 Schuhmacher P, Kim E, Hahn F, et al. Growth characteristics and therapeutic decision markers in von Hippel-Lindau disease patients with renal cell carcinoma. Orphanet $J$ Rare Dis 2019;14:235.

32 Motzer RJ, Escudier B, McDermott DF, et al. Nivolumab versus everolimus in advanced renal-cell carcinoma. $N$ Engl J Med 2015;373:1803-13.

33 Janjigian YY, Bendell J, Calvo E, et al. CheckMate-032 study: efficacy and safety of nivolumab and nivolumab plus ipilimumab in patients with metastatic esophagogastric cancer. J Clin Oncol 2018;36:2836-44.

34 Huang AC, Postow MA, Orlowski RJ, et al. T-Cell invigoration to tumour burden ratio associated with anti-PD-1 response. Nature 2017;545:60-5.

35 Brenner E, Schörg BF, Ahmetlić F, et al. Cancer immune control needs senescence induction by interferon-dependent cell cycle regulator pathways in tumours. Nat Commun 2020;11:1335.

36 Patel SP, Kurzrock R. Pd-L1 expression as a predictive biomarker in cancer immunotherapy. Mol Cancer Ther 2015;14:847-56.

37 Wang Q, Liu F, Liu L. Prognostic significance of PD-L1 in solid tumor: an updated meta-analysis. Medicine 2017;96:e6369.

38 Topalian SL, Hodi FS, Brahmer JR, et al. Safety, activity, and immune correlates of anti-PD-1 antibody in cancer. $N$ Engl J Med 2012;366:2443-54

39 Larkin J, Chiarion-Sileni V, Gonzalez R, et al. Combined nivolumab and ipilimumab or monotherapy in untreated melanoma. $N$ Engl J Med 2015;373:23-34.

40 Taube JM, Young GD, McMiller TL, et al. Differential expression of Immune-Regulatory genes associated with PD-L1 display in melanoma: implications for PD-1 pathway blockade. Clin Cancer Res 2015;21:3969-76.

41 Collins VP, Loeffler RK, TIVEY H. Observations on growth rates of human tumors. Am J Roentgenol Radium Ther Nucl Med 1956;76:988-1000. 
42 Joseph WL, Morton DL, Adkins PC. Variation in tumor doubling time in patients with pulmonary metastatic disease. J Surg Oncol 1971;3:143-9.

43 SCHWARTZ M. A biomathematical approach to clinical tumor growth. Cancer 1961;14:1272-94.

44 Gerlee P. The model muddle: in search of tumor growth laws. Cancer Res 2013;73:2407-11.

45 Benzekry S, Lamont C, Beheshti A, et al. Classical mathematical models for description and prediction of experimental tumor growth. PLoS Comput Biol 2014;10:e1003800.

46 Arai T, Kuroishi T, Saito Y, et al. Tumor doubling time and prognosis in lung cancer patients: evaluation from chest films and clinical follow- up study. Japanese lung cancer screening Research Group. Jpn J Clin Oncol 1994;24:199-204.

47 Usuda K, Saito Y, Sagawa M, et al. Tumor doubling time and prognostic assessment of patients with primary lung cancer. Cancer 1994;74:2239-44.

48 Murphy $\mathrm{H}$, Jaafari $\mathrm{H}$, Dobrovolny HM. Differences in predictions of ODE models of tumor growth: a cautionary example. BMC Cancer 2016;16:163.

49 Grob JJ, Long GV, Schadendorf D, et al. Disease kinetics for decision-making in advanced melanoma: a call for scenario-driven strategy trials. Lancet Oncol 2015;16:e522-6. 